\title{
A 10 años del inicio de la revista Fronteras en Medicina, un relanzamiento
}

\author{
10 years after the beginning of the Frontiers in Medicine \\ magazine, a relaunch
}

Fronteras en Medicina 20 I8; 13(3): | 27- 130. Doi: I0.3 | 954/RFEM/20 I803/0 I27-0 I 30

\section{PARECE QUE FUERA AYER}

Parece que fuera ayer cuando el Dr. José Amadeo Álvarez (Jefe de Hemodinamia) propusiera que la Revista Fronteras del intervencionismo endovascular (Figura 1) continuara con el nombre elegido por todos Fronteras en Medicina naciendo así su primer número en diciembre del 2008 (Figura 2). A pesar de que podría verse en la elección del nombre "fronteras" un límite, el sentido de elegirlo fue el de avanzada, el de desafiar permanentemente dichos límites a través de la producción de conocimiento. De esta manera se dio a luz una publicación que se edita a intervalos regulares, representando un aporte científico y cultural de inestimable valor para la Institución. Esta publicación es producto de la continuidad en el tiempo y expresión de un gran impulso del ser humano: el ver publicada sus ideas, como diría el notable escritor Oscar Wilde "El ver publicados nuestros sueños".

Las revistas científicas que se publican en nuestro país y en el exterior han fijado, y seguirán fijando, un papel de primer orden en la difusión del conocimiento médico general y de las diferentes especialidades. Sin embargo, de manera frecuente este tipo de publicaciones incursiona poco en los temas de educación médica, tanto de posgrado como de pregrado; y tiene poco acceso al conglomerado estudiantil. Estos dos aspectos primordiales son ampliamente cubiertos por nuestra Revista, lo cual le da un espectro temático muy amplio y de gran interés para profesores e investigadores, así como para estudiantes de las distintas Cátedras que integran las Universidades (UCA, UBA) aliadas a nuestro Hospital Universitario, tanto las de ciencias básicas, como las de ciencias clínicas, en sus distintos niveles y estructuras temáticas.

Otra faceta importante para destacar es que, entre las metas de la revista, se debe auspiciar la participación estudiantil en los trabajos de investigación para que así, los estudiantes se vayan familiarizando con la concisión y la precisión, así como para darse cuenta de las dificultades que encierra la investigación y la comunicación de un trabajo científico. Los estudiantes que publican de manera frecuente son los de la Cátedra de Historia de la Medicina en el cuarto año de la Carrera de Medicina de la UCA.

Por consiguiente, la Revista es, en síntesis, un órgano de altísima importancia para la divulgación científica y en particular para hacer del conocimiento el producto de la investigación autóctona, y de esta manera se convierte en un instrumento fundamental para la educación médica en nuestro Hospital ${ }^{1}$.

\section{NUESTRA HISTORIA}

Se inició allá por 2008 con un ejemplar en diciembre de dicho año. A partir de entonces se ha mantenido con cuatro números anuales. Estas páginas tuvieron plumas ilustres como las de los Dres. Alberto Agrest, Guillermo Jaim Echeverry, Arturo R. Rolla, Juan D. C. Emery, Juan C. Barreira y Juan D. Humphreys, entre otros. Durante el tiempo de su existencia ha sufrido algunas interrupciones en el transcurso de los años (pasar de papel a electrónico solo durante los años 2013 y 2014), pero ha superado con nuevos bríos y animada de la mejor buena fe de sus excelentes miembros de la Comisión Redactora que han asumido la responsabilidad de conducirla hacia el futuro. Estos diez años de nuestra revista brindan una ocasión propicia para felicitar a sus editores y colaboradores de ayer y hoy. Gracias a ellos este sueño hoy es una realidad, tenemos el reto de renovar. 


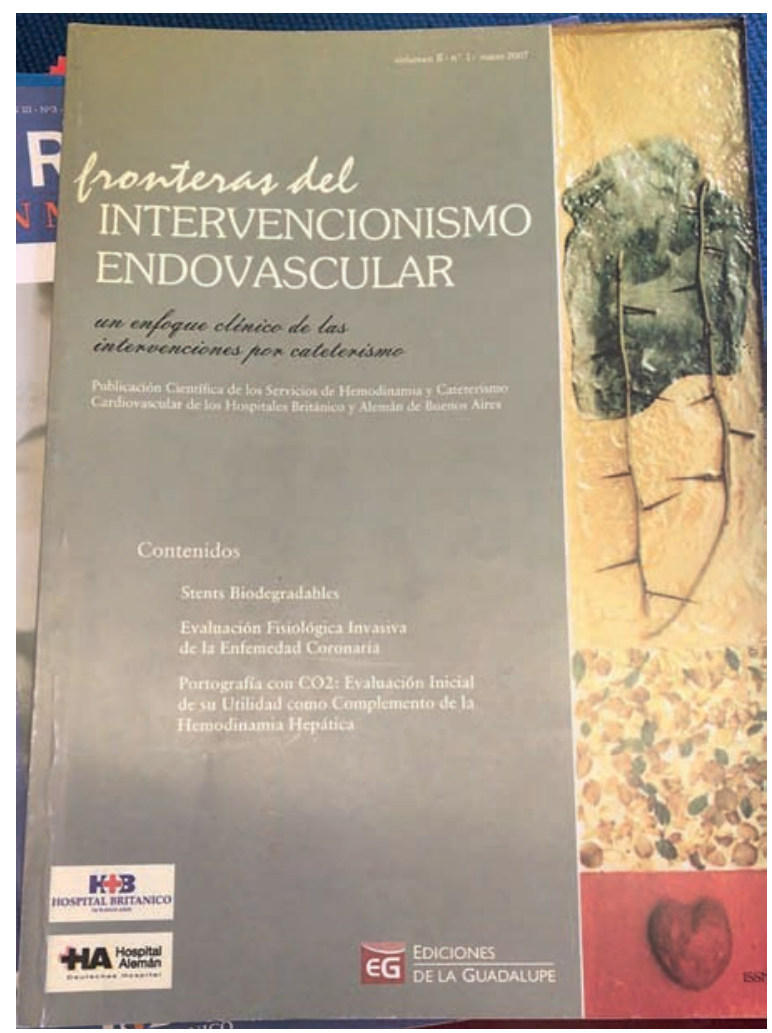

Figura I. Tapa de la Revista Fronteras del Intervencionismo Endovascular.

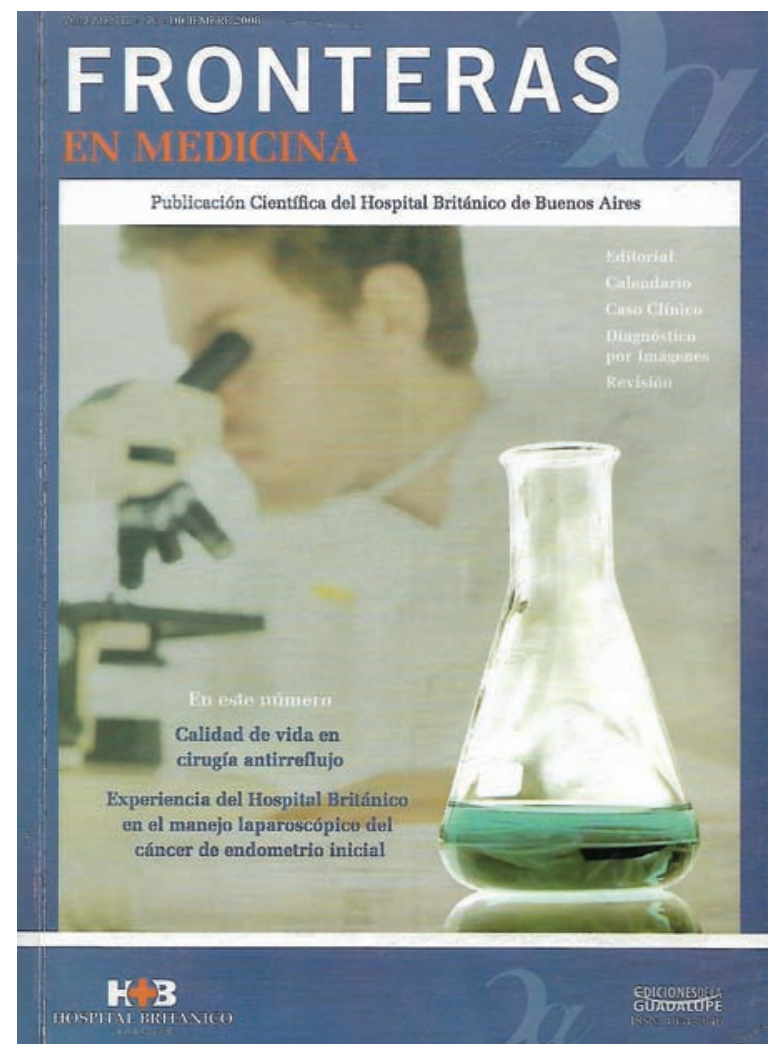

Figura 2. Tapa de la primera Revista Fronteras en Medicina.

\section{NUESTRA ANTECESORA}

La antecesora de la Revista Fronteras en Medicina fue la Revista Médica del Hospital Británico fue fundada en 1944 (Figura 3) ${ }^{2}$. En el primer número de 1944 escribió, entre otros, Eduardo E. Krapf "Accidentes y operaciones como expresión de tendencias auto-destructivas". En el segundo número de 1945 se pueden leer los trabajos de R. O. Comotti y Samuel Stuart Pennington sobre "Tumores del cuello (laringoceles)"; Monde H. Zommer sobre "A propósito de un caso de Kala azar"; Horacio A. Podestá escribió "Formas de comienzo cerebral del cáncer broncopulmonar"; Alberto E. Laurence describió "Divertículo gástrico en una diverticulosis múltiple del tubo digestivo". Los redactores (editores) eran: Manuel S Gullerto, Edgardo Horton-Greeves, Federico van Domselaar y Hugo F. Warneford-Thomson. La secretaria fue la Sra. R. de Michelson.

\section{EL NACIMIENTO DE LAS REVISTAS MÉDICAS}

Las primeras publicaciones periodísticas aparecieron en Europa en el curso del siglo XVII y entre las primeras estuvieron las destinadas al campo de la erudición en general. Se contaba con la revista inglesa de nombre Philosophical Transactions of the Royal Society of London (Figura 4), que apareció en el año 1665 y en la cual se publicaron las primeras "Memorias" sobre tópicos diversos. Se debió a la iniciativa del gran William Heberden (1702-1801), figura médica de gran relevancia en el transcurso del siglo XVIII, la creación de la revista denominada Medical Transactions (Trabajos Médicos) (Figura 5). El Dr. Heberden tiene un sitial en la Historia de la Medicina por haber escrito en forma magistral el cuadro clínico del "Angina de Pecho" que se publicó en esa revista. Previamente a la revista iniciada por Heberden, se había creado en la calle Fleet, Londres, el 17 de junio de 1784 Medicina Curiosa, que fue la primera revista en inglés con artículos solo de medicina editada por Thomas Basset (Figura 6).

\section{EL RELANZAMIENTO}

La Revista Fronteras, como órgano profesional, aspira a la información y divulgación de las actividades científicas e investigativas del Hospital Británico de Buenos Aires. Se está trabajando arduamente para lo- 


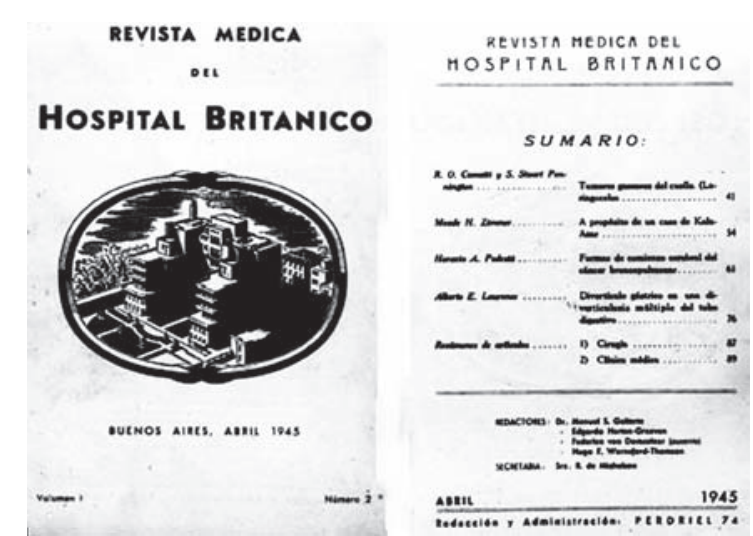

Figura 3. Revista Médica del Hospital Británico.
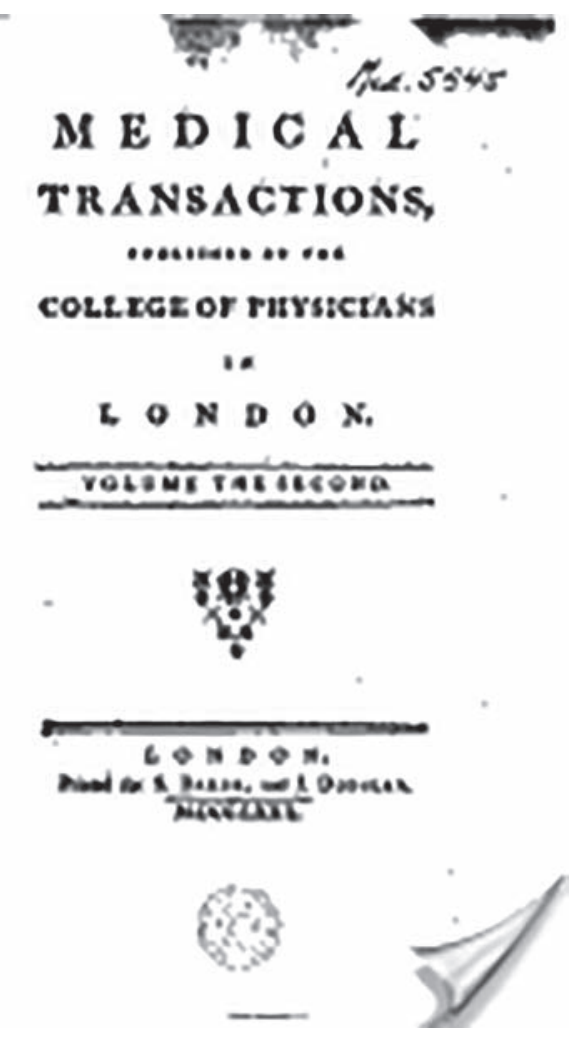

Figura 5. Medical Transactions. camente por el Hospital. Si bien al principio estuvimos juntos a Ediciones de la Guadalupe, desde hace años caminamos en armonía con Publicaciones Latinoamericanas, que es la editorial que nos asiste.

Desde hace un año se ha incorporado el Dr. Juan D. C. Emery quien se encarga de la revisión última del inglés; se ha mejorado la imagen de la revista a través de los aportes de la Dirección de Relaciones Institucionales y se ha perfeccionado la estrategia de comunicación por lo trabajado en conjunto con la Dirección de Procesos y Tecnología. Desde este número contamos con un International Standard Serial Number (ISSN) renovado, número con el cual nuestra revista está inscripta en bases internacionales; y Digital Object Identifier (DOI) para que nuestros trabajos tengan un número único de identificación a nivel mundial.

En una publicación de este tipo debe haber un gran equilibrio entre trabajos portadores de experiencias prácticas y los dedicados a comunicar planteamientos pedagógicos, así como actualizaciones en medicina general, en semiología, en clínica médica, quirúrgica, pediátrica y obstétrica, en salud pública y en medicina preventiva y social. 


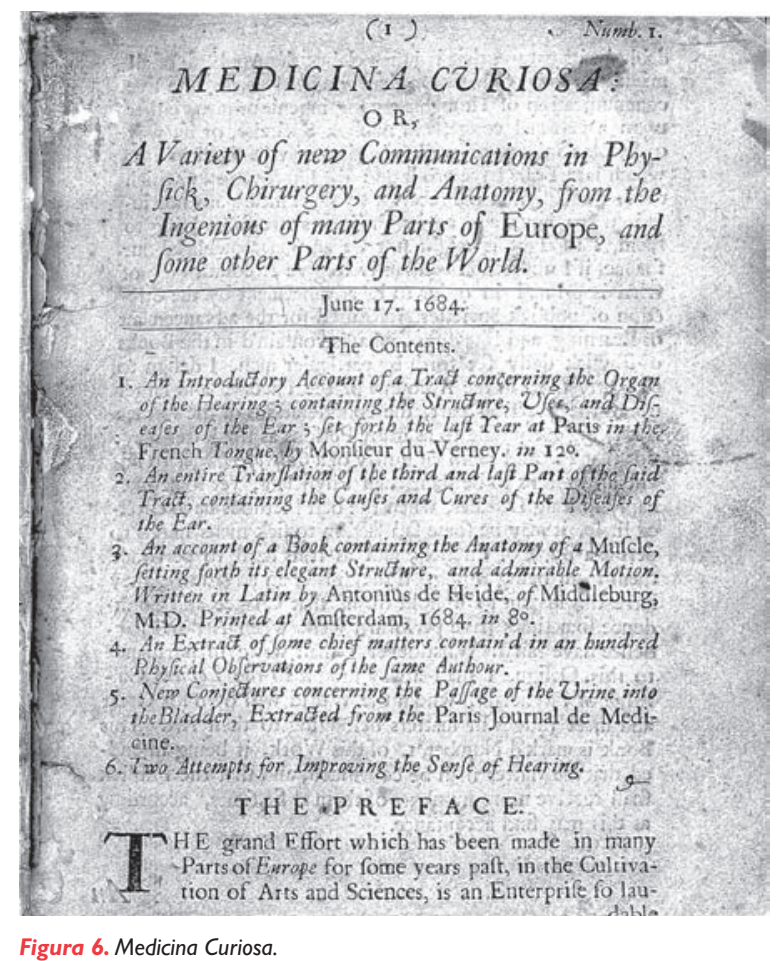

Figura 6. Medicina Curiosa.

Es difícil que una temática tan diversa se desarrolle en una sola publicación, sin perder unidad. El lograr este difícil equilibrio han sido factores esenciales la correcta selección del equipo editorial.

Delicada misión es la de presentar resultados, compartir preocupaciones y confrontar dudas con la opinión de quienes no están imbuidos en la cotidianidad de la Institución; constituye un ejercicio mental que esclarece el camino y afinará la dirección de sus pasos. Ello es especialmente cierto, por cuanto sus lectores serán profesionales calificados y estudiantes comprometidos con el trabajo científico.

La Revista tiene la finalidad de divulgar conocimientos y experiencias y contribuye a que la ciencia médica Argentina evolucione constantemente de manera amplia; al mismo tiempo, obliga a que las investigaciones se desarrollen sobre bases de inconmovible verdad.

Ignacio Chávez fue un cardiólogo mexicano líder en América de la transición de la cardiología antigua a la moderna. Él mencionó en el discurso pronunciado con motivo de la inauguración del Instituto Nacional de Cardiología de México, en 1944, acerca del tema "Sobre la Ciencia": "Necesitamos hacer ciencia, crear ciencia nosotros mismos y no pasarnos la vida repitiendo las verdades y errores que nos legaron otros. Mientras no hagamos eso, seremos los eternos ignorantes en el mundo de la ciencia. Si no tenemos tradición científica, si en la ciencia más que en nada no existe el mañana sino el ayer, comenzaremos por convertirnos nosotros en el ayer, para que tengan un mañana los que nos siguen"”.

Quiero invitar a todos a publicar. El equipo de la revista está abierto a todas las tendencias. El trabajo constante es la clave del éxito. Termino con un pensamiento de Bertolt Brecht: "Hay hombres que luchan un día y son buenos; hay otros que luchan un año y son mejores; hay quienes luchan muchos años y son muy buenos. Pero hay los que luchan toda la vida: esos son imprescindibles".

Pablo Young

Servicio de Clínica Médica, Hospital Británico de Buenos Aires, Rep. Argentina Email: pabloyoung2003@yahoo.com.ar

\section{BIBLIOGRAFÍA}

I. Young P.Acerca de las publicaciones. Fronteras en Medicina 2013:4:1 I 5-8.

2. Gotta C, Buzzi AE. Samuel Stuart Pennington y la Batalla del Río de la Plata. Primera Parte. Revista Argentina de Radiología 2007;71:387-93.

3. Humphreys JD, Young P. Paul Hamilton Wood (1907-1962). El máximo exponente de la cardiología clínica Británica del siglo XX Rev Med Chile 2012;140:121-4 